\title{
Comparison of mean squared error estimators under the Fay-Herriot model: application to poverty and percentage of food expenditure data
}

\author{
Yegnanew A. Shiferaw* (D), Jacqueline S. Galpin (D) \\ Department of Statistics, University of Johannesburg, Auckland Park Kingsway Campus, PO Box 524, \\ Johannesburg 2006, South Africa
}

\begin{abstract}
Small area estimates have received much attention from both private and public sectors due to the growing demand for effective planning of health services, apportioning of government funds and policy and decision making. The uncertainty of empirical best linear unbiased predictor (EBLUP) estimates is widely assessed by mean squared error (MSE). MSEs are criticized as they are not area specific since they do not depend on the direct estimators from the survey. In this paper, we compare the performances of different MSE estimators with respect to the relative bias and relative risk using a Monte Carlo simulation study. Simulation results suggest the superiority of the proposed MSEs over the existing methods in some situations. As a case study, the 2010/11 household consumption expenditure survey (HCES) and the 2007 housing and population census of Ethiopia have been used to study the performances of the MSE estimators.
\end{abstract}

Mathematics Subject Classification (2010). 62D05

Keywords. area-specific, EBLUP estimator, Fay-Herriot model, MSE estimator

\section{Introduction}

Reliable small area statistics are needed for local planning, formulating policies and programs, marketing decisions, allocation of funds and so on. For example, government policies and private sectors in both developed and developing countries increasingly demand poverty and income estimates for small areas [20]. The census data gives demographic information once in a decade, while sample surveys aim to provide estimates at the national or regional/provincial levels and pay little or no attention to the sub-national or district levels. Because planning large sample surveys with adequate sample size is expensive and time-consuming $[2,20]$. The direct survey estimates (estimates from the given sample survey) for small areas have unacceptably large standard errors and coefficient of variations (CV) due to small sample sizes. Therefore, it requires strengthening of such estimates with the use of information from census or other data sources from related small areas through linking models. This can be achieved by employing indirect small area estimators such as using the well known Fay-Herriot (FH) model [9] in small area estimation. The

\footnotetext{
*Corresponding Author.

Email addresses: yegnanews@uj.ac.za (Y. A. Shiferaw), jacky@galpin.co.za (J. S. Galpin)

Received: 23.01.2019; Accepted: 06.01.2020
} 
FH model was introduced by [9] to obtain model-based estimates for per-capita income for small places in the United States. This model is useful in cases where auxiliary data are available at the area level or when it is not possible to link the information of the sample units with census data or unit-level data, which might not be available due to confidentiality issues $[17,20]$.

Small area estimation has been extensively used as an indirect estimation technique for estimating poverty indicators, per-capita income, etc. Moreover, indirect estimation of small area means or totals through linking models has received much attention in recent years, including mean squared error (MSE) [7]. The variability of indirect small area estimators is often measured using MSE. According to [20], MSE estimators are areaspecific in the sense that the MSE depends on the area-specific data, that is, on the direct estimator $y_{i}$, if we are estimating in the area $i$. This is the sense of the term area-specific used in [20]. The estimated MSE obtained by [17] or [5] are not area-specific since the MSEs do not depend directly on the direct estimator $y_{i}$. For this reason, [19] proposed alternative area-specific MSEs to fill this gap. Moreover, [22] proposed confidence intervals using some area-specific MSEs. The MSE estimators by [19] are unstable, but its effect should be small for a large number of small areas $(\mathrm{m})[20]$. The aims of this paper are: (i) to study and compare the performance of the conventional MSEs $[5,17]$ and the areaspecific MSEs [19,22] in terms of RB and RR through a simulation study; and (ii) to provide the estimates of percentage of food expenditure and poverty measure at the zone level for the Ethiopian data and to evaluate the results based on the RB, RR and width of prediction intervals.

\section{Materials and methods}

\subsection{The Fay-Herriot model}

The well-known FH small area estimation model [9] consists of two levels. Level 1 represents the sampling model

$$
y_{i} \mid \theta_{i} \stackrel{i n d}{\sim} N\left[\theta_{i}, \psi_{i}\right], i=1, \ldots, m,
$$

where $\psi_{i}$ represents the sampling variance of $y_{i}$ to be estimated from the sample survey. Level 2 represents the linking model,

$$
\theta_{i} \stackrel{i n d}{\sim} N\left[\mathbf{x}_{\mathbf{i}}^{\prime} \boldsymbol{\beta}, A\right], i=1, \ldots, m
$$

Level 1 accounts the sampling variability of the direct survey estimates $y_{i}$ of the true small area means $\theta_{i}$. Level 2 is set to link $\theta_{i}$ to a $p \times 1$ vector of auxiliary variables $\mathbf{x}_{i}=\left(x_{i 1}, \cdots, x_{i p}\right)^{\prime}$. Let $y_{1}, \cdots, y_{m}$ be observations for the $m$ small areas. Then the $\mathrm{FH}$ model is defined as

$$
y_{i}=\mathbf{x}_{\mathbf{i}}^{\prime} \boldsymbol{\beta}+v_{i}+e_{i}, i=1, \cdots, m,
$$

where

- $\boldsymbol{\beta}=\left(\beta_{1}, \ldots, \beta_{p}\right)^{\prime}$ is a $p \times 1$ vector of unknown regression coefficients,

- $v_{i}$ is the area-specific random effects with $E\left(v_{i}\right)=0$ and $\operatorname{Var}\left(v_{i}\right)=A(\geq 0)$,

- $e_{i}$ is the sampling error with $E\left(e_{i}\right)=0$ and $\operatorname{Var}\left(e_{i}\right)=\psi_{i}$, is assumed to be known for all areas $i=1,2, \cdots, m$,

- $v_{i}^{\prime} s$ and $e_{i}^{\prime} s$ are independent with $v_{i} \stackrel{i i d}{\sim} N(0, A)$ and $e_{i} \stackrel{i n d}{\sim} N\left(0, \psi_{i}\right)$.

The FH model is designed to estimate the small area means $\theta_{i}=\mathbf{x}_{\mathbf{i}}^{\prime} \boldsymbol{\beta}+v_{i}, 1, \ldots, m$. Letting $\mathbf{X}=\left(x_{1}, \ldots, x_{m}\right)^{\prime}, \mathbf{y}=\left(y_{1}, \ldots, y_{m}\right)^{\prime}, \mathbf{v}=\left(v_{1}, \ldots, v_{m}\right)$ and $\mathbf{e}=\left(e_{1}, \ldots, e_{m}\right)$, then the matrix natation of the FH model may be expressed as

$$
\mathbf{y}=\mathbf{X} \boldsymbol{\beta}+\mathbf{v}+\mathbf{e} .
$$


The variance-covariance matrix of $\mathbf{y}$ is $\boldsymbol{\Sigma}(A)=\operatorname{diag}\left(\psi_{1}, \ldots, \psi_{m}\right)+A \mathbf{I}_{m}$, where $\mathbf{I}_{m}$ denotes an $m \times m$ identity matrix.

In practice, there are different procedures for the estimation of unknown model parameters $\boldsymbol{\beta}$ and $A$. The FH method of moment estimator by [9], maximum likelihood (ML) and restricted maximum likelihood (REML) methods by [5], and so on. Substituting $\hat{A}$ for $A$ reveals the empirical best linear unbiased predictor (EBLUP) given by

$$
\hat{\theta}_{i}^{\mathrm{EB}}=\left(1-\hat{\gamma}_{i}\right) y_{i}+\hat{\gamma}_{i} \mathbf{x}_{\mathbf{i}}^{\prime} \hat{\boldsymbol{\beta}}, \quad i=1, \ldots, m,
$$

where $\hat{\boldsymbol{\beta}}=\left(\mathbf{X}^{\prime} \hat{\boldsymbol{\Sigma}}^{-\mathbf{1}} \mathbf{X}\right)^{-\mathbf{1}} \mathbf{X}^{\prime} \hat{\boldsymbol{\Sigma}}^{-\mathbf{1}} \mathbf{y}=\left\{\sum_{i=1}^{m}\left(\hat{A}+\psi_{i}\right)^{-1} \mathbf{x}_{\mathbf{i}} \mathbf{x}_{\mathbf{i}}^{\prime}\right\}^{-1} \sum_{i=1}^{m}\left(\hat{A}+\psi_{i}\right)^{-1} \mathbf{x}_{\mathbf{i}} y_{i}$ is the weighted least squares (WLS) estimator of $\boldsymbol{\beta}$ and $\hat{\gamma}_{i}=\frac{\psi_{i}}{\psi_{i}+\hat{A}}$, the weight attached to the regression synthetic estimator. The EBLUP is a weighted combination of a direct survey estimator $y_{i}$ and the regression-synthetic estimator $\mathbf{x}_{\mathbf{i}}^{\prime} \hat{\boldsymbol{\beta}}$. When $\psi_{i}$ is small relative to $\hat{A}+\psi_{i}$, then the direct estimator $y_{i}$ is efficient. However, when $\psi_{i}$ is large as compared to $\hat{A}+\psi_{i}$, then more weight is attached to the regression synthetic estimator $\mathbf{x}_{\mathbf{i}}^{\prime} \hat{\boldsymbol{\beta}}[16]$.

\subsection{Mean squared error}

The MSE is the most commonly used measures of uncertainty of $\hat{\theta}_{i}^{\mathrm{EB}}$ in small area estimation. A second-order correct approximation for the MSE of EBLUP has been obtained first by [17] using a moment estimator $A$. Later, [5] derived a second order correct approximation of the MSE of EBLUP for the ML and REML estimators of $A$. A detailed review of EBLUP and MSE are given in [20]. The MSE of $\hat{\theta}_{i}^{\mathrm{EB}}$ is defined as

$$
\operatorname{MSE}\left(\hat{\theta}_{i}^{\mathrm{EB}}\right)=E\left\{\hat{\theta}_{i}^{\mathrm{EB}}-\theta_{i}\right\}^{2}
$$

The MSE of $\hat{\theta}_{i}^{\mathrm{EB}}$ which is accurate to the order $O\left(\mathrm{~m}^{-1}\right)$ can be approximated by

$$
\operatorname{MSE}\left(\hat{\theta}_{i}^{\mathrm{EB}}\right)=g_{1 i}(A)+g_{2 i}(A)+g_{3 i}(A)+O\left(m^{-1}\right),
$$

where $g_{1 i}(A)=\frac{A \psi_{i}}{A+\psi_{i}}, \quad g_{2 i}(A)=\gamma_{i}^{2} \mathbf{x}_{i}^{\prime}\left(\mathbf{X}^{\prime} \boldsymbol{\Sigma}^{-1} \mathbf{X}\right)^{-1} \mathbf{x}_{i}$ and $g_{3 i}(A)=\frac{\psi_{i}^{2}}{\left(A+\psi_{i}\right)^{3}} V(\hat{A})+o\left(m^{-1}\right)$ (see $[17,20])$. For example, Datta et al. (2002) derived the asymptotic variance of the FH moment estimator which is given by $V(\hat{A})=2 m\left[\sum_{i=1}^{m} \frac{1}{A+\psi_{i}}\right]^{-2}$.

For the REML estimator of $A$, [5] obtained an unbiased estimator of the MSE of $\hat{\theta}_{i}^{\mathrm{EB}}$ given by

$$
\widehat{\operatorname{MSE}}\left(\hat{\theta}_{i}^{\mathrm{EB}}\right)=g_{1 i}\left(\hat{A}_{R E}\right)+g_{2 i}\left(\hat{A}_{R E}\right)+2 g_{3 i}\left(\hat{A}_{R E}\right)
$$

where $g_{1 i}\left(\hat{A}_{R E}\right)=\hat{A}_{R E} \psi_{i}\left(\hat{A}_{R E}+\psi_{i}\right)^{-1}, g_{2 i}\left(\hat{A}_{R E}\right)=\frac{\psi_{i}^{2}}{\left(\hat{A}_{R E}+\psi_{i}\right)^{2}} \mathbf{x}_{i}^{\prime}\left\{\sum_{u=1}^{m}\left(\hat{A}_{R E}+\psi_{u}\right)^{-1} \mathbf{x}_{u} \mathbf{x}_{u}^{\prime}\right\}^{-1} \mathbf{x}_{i}$, $g_{3 i}\left(\hat{A}_{R E}\right)=\hat{\gamma}_{i}^{2} \frac{V\left(\hat{A}_{R E}\right)}{\hat{A}_{R E}+\psi_{i}}$ and $V\left(\hat{A}_{R E}\right)=\frac{2}{\sum_{i=1}^{m}\left(\hat{A}_{R E}+\psi_{i}\right)^{-2}}$. It is known that the $\widehat{\operatorname{MSE}}\left(\hat{\theta}_{i}^{\mathrm{EB}}\right)$ is second order unbiased if $E\left[\widehat{\operatorname{MSE}}\left(\hat{\theta}_{i}^{\mathrm{EB}}\right)\right]=\operatorname{MSE}\left(\hat{\theta}_{i}^{\mathrm{EB}}\right)+O\left(m^{-1}\right)$ (see [20]).

For the ML estimator of $A$, [5] obtained an unbiased estimator of the MSE of $\hat{\theta}_{i}^{\mathrm{EB}}$ given by

$$
\widehat{\operatorname{MSE}}_{M L}\left(\hat{\theta}_{i}^{\mathrm{EB}}\right)=g_{1 i}\left(\hat{A}_{M L}\right)+g_{2 i}\left(\hat{A}_{M L}\right)+2 g_{3 i}\left(\hat{A}_{M L}\right)-b_{\hat{A}_{M L}}\left(\hat{A}_{M L}\right) \nabla g_{1 i}\left(\hat{A}_{M L}\right),
$$

where $\nabla g_{1 i}\left(\hat{A}_{M L}\right)=\left(\frac{\psi_{i}}{\hat{A}_{M L}+\psi_{i}}\right)^{2}>0$. The estimated bias of $\hat{A}_{M L}$ for the ML estimator $\hat{A}_{M L}$ is given by

$$
b_{\hat{A}_{M L}}\left(\hat{A}_{M L}\right)=\frac{-\operatorname{tr}\left\{\left[\sum_{u=1}^{m}\left(\hat{A}+\psi_{u}\right)^{-1} \mathbf{x}_{u} \mathbf{x}_{u}^{\prime}\right]^{-1}\left[\sum_{u=1}^{m}\left(\hat{A}_{M L}+\psi_{u}\right)^{-2} \mathbf{x}_{u} \mathbf{x}_{u}^{\prime}\right]\right\}}{\sum_{u=1}^{m}\left(\hat{A}_{M L}+\psi_{u}\right)^{-2}}
$$


Similarly, for the FH estimator of $A$, [6] obtained an unbiased estimator of the MSE of $\hat{\theta}_{i}^{\mathrm{EB}}$ given by

$$
\widehat{\operatorname{MSE}}_{F H}\left(\hat{\theta}_{i}^{\mathrm{EB}}\right)=g_{1 i}\left(\hat{A}_{F H}\right)+g_{2 i}\left(\hat{A}_{F H}\right)+2 g_{3 i}\left(\hat{A}_{F H}\right)-b_{\hat{A}_{F H}}\left(\hat{A}_{F H}\right)\left\{\gamma_{i}\left(\hat{A}_{F H}\right)\right\}^{2} .
$$

The bias to terms of order $O\left(\mathrm{~m}^{-1}\right)$ for the $\mathrm{FH}$ model is given by

$$
b_{\hat{A}_{F H}}\left(\hat{A}_{F H}\right)=\frac{2\left\{m \sum_{u=1}^{m}\left(\hat{A}_{F H}+\psi_{u}\right)^{-2}-\left\{\sum_{u=1}^{m}\left(\hat{A}_{F H}+\psi_{u}\right)^{-1}\right\}^{2}\right\}}{\sum_{u=1}^{m}\left(\hat{A}_{F H}+\psi_{u}\right)^{-2}} .
$$

\subsection{Area-specific mean squared errors}

According to [20], MSE estimators are area-specific when they incorporate area-specific direct survey estimators within it. The $g_{3 i}(A)$ which was proposed by $[5,17]$ and others are given by

$$
g_{3 i}(A)=\frac{\psi_{i}^{2}}{\left(A+\psi_{i}\right)^{3}} V(\hat{A}) .
$$

This $g_{3 i}(A)$ is not area-specific since it does not incorporate the direct survey estimate $y_{i}$ within it. Thus, [19] and [22] proposed alternative area-specific $g_{3 i}\left(A, y_{i}\right)^{\prime} s$ under the FH model. The [19] $g_{3 i}\left(A, y_{i}\right)$ is given as

$$
\text { Rao : } \quad \tilde{g}_{3 i}\left(A, y_{i}\right)=\frac{\psi_{i}^{2}}{\left(\psi_{i}+A\right)^{4}}\left(y_{i}-\mathbf{x}_{i}^{\prime} \tilde{\boldsymbol{\beta}}\right)^{2} V(\hat{A}) .
$$

Similarly, [22] proposed the following alternative area-specific $g_{3 i}\left(A, y_{i}\right)^{\prime} s$ as follows:

$$
\mathrm{JY} \quad: \quad g_{3 \mathrm{JY} i}\left(A, y_{i}\right)=\frac{\psi_{i}^{2}}{\left(\psi_{i}+A\right)^{4}} \frac{\left(y_{i}-\mathbf{x}_{i}^{\prime} \tilde{\boldsymbol{\beta}}\right)^{2}}{\mathrm{~V}\left(y_{i}-\mathbf{x}_{i}^{\prime} \tilde{\boldsymbol{\beta}}\right)} V(\hat{A}),
$$

where $\mathrm{V}\left(y_{i}-\mathbf{x}_{i}^{\prime} \tilde{\boldsymbol{\beta}}\right)=A+\psi_{i}-\mathbf{x}_{i}^{\prime}\left(\mathbf{X}^{\prime} \boldsymbol{\Sigma}^{-1} \mathbf{X}\right)^{-1} \mathbf{x}_{i}$.

The second $g_{3 i}(A)^{\prime} s$ which was proposed by [22] has the form:

$$
\text { JY1 : } \quad \tilde{g}_{3 J Y 1 i}(A)=g_{3 i}(A)-g_{5 i}(A),
$$

where $g_{5 i}(A)=\frac{g_{2 i}(A)}{\left(A+\psi_{i}\right)^{2}} V(\hat{A})$. Note that $\tilde{g}_{3 J Y 1 i}(A)$ in $(2.14)$ does not include $y_{i}$, but it includes the area-specific auxiliary variables unlike the usual $g_{3 i}(A)$ in equation (2.11). Equation (2.13) standardizes the residuals in order to make them scale-free [1].

\section{Results and discussion}

\subsection{Comparison of the MSEs}

We now investigate the finite sample performances of the different MSEs under the FH model. Note that MSE_Rao, MSE_JY and MSE_JY1 are the MSE estimators based on (2.12), (2.13) and (2.14) respectively under the three different methods of estimation. However, MSE_REML, MSE_ML and MSE_FH are the MSE estimators based on (2.11) under the REML, ML and FH methods of estimation [24]. The MSEs we want to compare are given as follows. The four different MSE estimators based on the REML estimator ([5]) are given by:

$$
\begin{aligned}
& \operatorname{MSE} \_R a o_{i} \approx g_{1 i}(\hat{A})+g_{2 i}(\hat{A})+g_{3 i}(\hat{A})+g_{3 \operatorname{Rao} i}^{*}\left(\hat{A}, y_{i}\right) \text {. } \\
& \mathrm{MSE}_{-} \mathrm{JY}_{i} \approx g_{1 i}(\hat{A})+g_{2 i}(\hat{A})+g_{3 i}(\hat{A})+g_{3 \mathrm{JY} i}^{*}\left(\hat{A}, y_{i}\right) \text {. } \\
& \text { MSE_JY1 } 1_{i} \approx g_{1 i}(\hat{A})+g_{2 i}(\hat{A})+g_{3 i}(\hat{A})+g_{3 J Y 1 i}^{*}(\hat{A}) \text {. } \\
& \operatorname{MSE} \_R E M L_{i} \approx g_{1 i}(\hat{A})+g_{2 i}(\hat{A})+2 g_{3 i}(\hat{A}) .
\end{aligned}
$$

The four different MSE estimators based on the ML ([5]) and FH ([6]) estimators can be written similar to the REML case. The relative bias $(\mathrm{RB})$ and relative risk $(\mathrm{RR})$ are 
used to compare the MSEs given above. Smaller RB and RR values indicate better MSE performance ([12]). The RB and RR are given as follows:

$\mathrm{RB}_{i}=100 \times \frac{E\left[\widehat{\operatorname{MSE}}\left(\hat{\theta}_{i}^{\mathrm{EB}}\right)-\operatorname{MSE}\left(\hat{\theta}_{i}^{\mathrm{EB}}\right)\right]}{\operatorname{MSE}\left(\hat{\theta}_{i}^{\mathrm{EB}}\right)}, \quad \operatorname{RR}_{i}=100 \times \frac{E\left\{\widehat{\operatorname{MSE}}\left(\hat{\theta}_{i}^{\mathrm{EB}}\right)-\operatorname{MSE}\left(\hat{\theta}_{i}^{\mathrm{EB}}\right)\right\}^{2}}{\left\{\operatorname{MSE}\left(\hat{\theta}_{i}^{\mathrm{EB}}\right)\right\}^{2}}$.

The average RB and RR of each MSE estimator over areas are given by

$$
\overline{R B}=\frac{1}{m} \sum_{i=1}^{m} \mathrm{RB}_{i}, \quad \overline{R R}=\frac{1}{m} \sum_{i=1}^{m} \mathrm{RR}_{i}, i=1, \cdots, m .
$$

In addition to $\mathrm{RB}$ and $\mathrm{RR}$, the coverage probability (CP) of prediction intervals by [17], [4] and [22] are used to compare the proposed MSEs. The confidence interval proposed by [4] is easy to construct, but it has under-coverage problem since it does not account the uncertainty due to the estimation of $\beta$ and $A$. This confidence interval is accurate to terms $O\left(\mathrm{~m}^{-1}\right)$, not accurate enough in most small area applications [26]. The [4] empirical Bayes interval for $\theta_{i}$ based on $\hat{\theta}_{i}^{E B}$ is given as follows:

$$
\mathrm{I}_{i}^{\mathrm{Cox}}: \hat{\theta}_{i}^{\mathrm{EB}} \pm z_{\alpha / 2} \psi_{i}^{1 / 2}\left(1-\hat{\gamma}_{i}\right)^{1 / 2} .
$$

Similar to [4]'s method, the confidence interval proposed by [17] is also accurate to terms $O\left(\mathrm{~m}^{-1}\right)$. The confidence interval [17] for $\theta_{i}$ can be written as follows:

$$
\mathrm{I}_{i}^{\mathrm{PR}}: \hat{\theta}_{i}^{\mathrm{EB}} \pm z_{\alpha / 2} \sqrt{\widehat{\mathrm{MSE}}\left(\hat{\theta}_{i}^{\mathrm{EB}}\right)} .
$$

The area specific corrected confidence intervals for a small area mean $\theta_{i} ; i=1, \cdots, m$ based on $\hat{\theta}_{i}^{E B}$ are proposed by [22] as follows:

$$
\mathrm{I}_{i}^{\mathrm{FH}}: \hat{\theta}_{i}^{\mathrm{EB}} \pm t_{\alpha}^{*} \sqrt{\widehat{\mathrm{MSE}}\left(\hat{\theta}_{i}^{\mathrm{EB}}\right)},
$$

where $t_{\alpha}^{*}=z_{\alpha / 2}(1+h(\hat{A})), h(\hat{A})=\left(z_{\alpha / 2}^{2}+1\right) \frac{\psi_{i}^{2}}{8 \hat{A}^{2}\left(\hat{A}+\psi_{i}\right)^{2}} V(\hat{A})$.

$$
\mathrm{I}_{i}^{\mathrm{Rao}}: \hat{\theta}_{i}^{\mathrm{EB}} \pm t_{\mathrm{Rao}} \sqrt{\mathrm{MSE} \_\mathrm{RaO}_{\mathrm{i}}},
$$

where $t_{\text {Rao }}=z_{\alpha / 2}+\left(z_{\alpha / 2}^{3}+z_{\alpha / 2}\right) \frac{\left(A+\psi_{i}\right) g_{3 \text { Rao }}}{8 A^{2}}$.

$$
\mathrm{I}_{i}^{\mathrm{JY}}: \hat{\theta}_{i}^{\mathrm{EB}} \pm t_{\mathrm{JY}} \sqrt{\mathrm{MSE} \_\mathrm{JY}},
$$

where $t_{\mathrm{JY}}=z_{\alpha / 2}+\left(z_{\alpha / 2}^{3}+z_{\alpha / 2}\right) \frac{\left(A+\psi_{i}\right) g_{3 \mathrm{JY} i}}{8 A^{2}}$.

$$
\mathrm{I}_{i}^{\mathrm{JY} 1}: \hat{\theta}_{i}^{\mathrm{EB}} \pm t_{\mathrm{JY} 1} \sqrt{\mathrm{MSE} \_\mathrm{JY} 1_{\mathrm{i}}},
$$

where $t_{\mathrm{JY} 1}=z_{\alpha / 2}+\left(z_{\alpha / 2}^{3}+z_{\alpha / 2}\right) \frac{\left(A+\psi_{i}\right) g_{3 \mathrm{JY} 1 i}}{8 A^{2}}$.

The performance of the above confidence intervals are evaluated using the $\mathrm{CP}$ given as follows:

$$
\mathrm{CP}_{i}=\sum_{i=1}^{m} \frac{\left|\theta_{i} \in \mathrm{CI}_{i}\right|}{m}
$$

where, $\mathrm{i}=1, \ldots, \mathrm{m}, \mathrm{CI}_{i}$ is the confidence interval of a certain method.

3.1.1. Simulation setup. The simulation experiments are carried out through considering the [6] with minor modifications. The range of small areas are selected through consideration of related literatures in the area. $m$ cannot be large in many applications [11]. Researchers such as [25] considered $m=30$ in testing the performance of their proposed step-wise Bayes estimator. Other researchers such as [3] considered a sample of small areas such as $m=8,20$ and 100 to assess their proposed measure of goodness-of-fit statistics. Another study by [14] considered a range of small areas $m=15$ and 45 to study the asymptotic behaviour of their proposed confidence intervals. Further, [22] considered a sample of small areas such as $m=15,20,30,40,50,60,80$ and 100 for comparing their 
proposed area specific confidence intervals under the FH model. Similarly [23] considered a sample of small areas such as $m=10,15,30,45$ and 60 in comparing their proposed confidence intervals. Taking the above literature into consideration and to study the effect of $m$, this paper considered a range of small areas such as $m=10,15,30,45$ and 60 . The vector of auxiliary variables are given as $\mathbf{x}_{i}=\left(1, \mathbf{x}_{i}\right)^{\prime}$, where $\mathbf{x}_{i} ; i=1, \ldots, m$ were generated i.i.d. from $N(0,1), \boldsymbol{\beta}=(1,1)^{\prime}$ and $A=1$. The sampling errors, $e_{i}$, were generated from $N\left(0, \psi_{i}\right)$ for $\psi_{i}$ specified by the following three different variance patterns: pattern (I) $0.7,0.6,0.5,0.4,0.3$, pattern (II) 2, 0.6, 0.5, 0.4, 0.2 and pattern (III) 4, 0.6, 0.5, $0.4,0.1$. The random effects, $v_{i}$ are generated from two different distributions, namely the normal $N(0,1)$ and the Laplace $(0,1)$ distributions. We have considered the Laplace distribution to assess the robustness of the methods to possible deviations from the normality assumptions. We considered two different cases; (i) without covariates: $x_{i} \boldsymbol{\beta}=0$, we generate 10,000 data sets from $y_{i}=v_{i}+e_{i},(i=1, \cdots, m)$ and (ii) with covariates: $\boldsymbol{\beta}=(1,1)^{\prime}$, we generate 10,000 data sets from $y_{i}=x_{i} \boldsymbol{\beta}+v_{i}+e_{i}, \quad(i=1, \cdots, m)$ to account for the estimation of the unknown regression parameters which comes from the real data applications. The SAS code by [13] were adopted with modification to check the performance of the proposed MSE estimators.

3.1.2. Simulation results without covariates. Following [6], we used a model with no auxiliary variables to obtain Table 1 . These results are shown separately for each of the sampling variances $\psi_{i}$, distinguishing among the cases $\hat{A}=\hat{A}_{M L}, \hat{A}=\hat{A}_{R E M L}$ and $\hat{A}=\hat{A}_{F H}$. The FH, ML and REML correspond to the FH, ML and REML estimators of the MSE of the EBLUP, respectively [24].

Table 1 shows the RB and RR values of the MSE estimators that were obtained through the simulation study for $m=15$ without covariates. $R R$ is a number that measures the risk of using MSE estimators under certain circumstances. The lower risk MSE estimators perform better than their higher risk counterparts. The RR of MSE_Rao, MSE_JY and MSE_JY1 are nearly zero for the ML, REML and FH methods for all patterns. In terms of RB and RR the MSE estimators based on the ML, REML and FH behave similarly for the pattern (I), with no particular one emerging as clearly better than the other three. The MSE estimators based on the FH method have a very small bias for all variance patterns. The bias of the MSE_JY1 estimator is smaller than MSE_Rao and MSE_JY for the ML and REML methods. The bias and risk of all the estimators are less than $7 \%$ when $\hat{A}=\hat{A}_{F H}$ for both patterns (II) and (III) are negligible for group G5 and the normal distribution. Under the Laplace distribution, the RR of MSE estimators based on the FH method are less than $7 \%$ in all cases [24].

3.1.3. Simulation results with covariates. For the FH model incorporating covariates, the RB and RR of MSE estimators are presented in Table 2. As shown in Table 2, for pattern (II), the average RR of MSE_ML, MSE_Rao, MSE_JY and MSE_JY1 are $6.32 \%, 1.22 \%, 1.66 \%$ and $1.38 \%$ respectively [24]. Figures $1-3$ plot the average RBs of the MSE estimators over the range of $m$. We have simulated the RB for the 5 groups within each pattern and $m$. Then we took the average of the absolute values of the RB over groups for each pattern and $m$. We have presented the findings for the patterns (I), (II) and (III) in Figures 1, 2 and 3 respectively, as shown below. The green, blue, red and black colours indicate that the MSE estimators based on $g_{3 i}(\hat{A})$ 's (Rao2001, JY, JY1 and ML or REML or FH) respectively [24]. The key findings in Figure 1 are:

- For the homogeneous $\psi_{i}$ pattern, the average RB of all the MSE estimators is less than $10 \%$.

- The average RB of MSE_Rao, MSE_JY and MSE_JY1 are less than the MSE of the ML and REML estimators. 
- All the MSE estimators based on the FH method are identical. They are nearly unbiased.

Table 1. Simulated values of the RB and RR of $\widehat{\operatorname{MSE}}\left(\hat{\theta}_{i}^{\mathrm{EB}}\right)$ for $A=1, m=15$, $\psi_{i}$ pattern (I), (II), (III) and for the normal and the Laplace random effects distributions

\begin{tabular}{|c|c|c|c|c|c|c|c|c|c|c|c|c|c|c|c|c|c|c|}
\hline \multicolumn{10}{|c|}{ Normal Distribution } & \multicolumn{9}{|c|}{ Laplace Distribution } \\
\hline \multirow{2}{*}{ Groups } & \multicolumn{3}{|c|}{ Pattern I } & \multicolumn{3}{|c|}{ Pattern II } & \multicolumn{3}{|c|}{ Pattern III } & \multicolumn{3}{|c|}{ Pattern I } & \multicolumn{3}{|c|}{ Pattern II } & \multicolumn{3}{|c|}{ Pattern III } \\
\hline & ML & $\mathrm{RE}$ & $\mathrm{FH}$ & ML & $\mathrm{RE}$ & FH & ML & $\mathrm{RE}$ & $\mathrm{FH}$ & ML & $\mathrm{RE}$ & $\mathrm{FH}$ & ML & $\mathrm{RE}$ & $\mathrm{FH}$ & ML & $\mathrm{RE}$ & FH \\
\hline \multicolumn{19}{|c|}{ RB } \\
\hline \multicolumn{19}{|c|}{ MSE based on ML, REML and FH methods } \\
\hline G1 & -12.4 & -7.7 & -0.8 & -15.4 & -8.5 & -2.9 & -15.6 & -6.9 & -4.8 & -16.7 & -9.1 & 1.9 & -20 & -9.5 & -3.8 & -21.9 & -7.5 & -9.4 \\
\hline G2 & -10.9 & -6.5 & 0.4 & -12.8 & -7.6 & -0.4 & -14.8 & -8.4 & -3.3 & -15.8 & -8.4 & 3.1 & -20 & -10.8 & -0.6 & -27.6 & -14.1 & -12.3 \\
\hline G3 & -11.4 & -7.2 & -0.6 & -13.2 & -8.3 & -1.3 & -15.3 & -9 & -4.6 & -14.5 & -7.6 & 4.3 & -19.2 & -10.2 & 0.3 & -27.4 & -13.7 & -12.9 \\
\hline G4 & -8.7 & -4.8 & 1.6 & -10.6 & -5.8 & 0.9 & -13.1 & -6.7 & -3 & -13.5 & -6.9 & 5.8 & -18.4 & -9.6 & 1.4 & -27.8 & -13.8 & -13.8 \\
\hline G5 & -7.2 & -3.8 & 2.4 & -6.5 & -2.7 & 3.5 & -10.5 & -1.5 & -2.5 & -9.4 & -3.2 & 10.9 & -9.5 & -0.7 & 16.7 & -23.8 & -1 & -1.5 \\
\hline \multicolumn{19}{|c|}{ MSE_Rao } \\
\hline G1 & -1.1 & -1.1 & -0.9 & -4 & -2.7 & -3 & -4.7 & -2.4 & -4.5 & 1.6 & 1.5 & 1.7 & -5.3 & -2.6 & -3.5 & -9.6 & -3 & -8.9 \\
\hline G2 & 0.5 & 0.3 & 0.3 & -0.5 & -0.1 & -0.3 & -3 & -1 & -3.3 & 3.3 & 2.8 & 2.8 & -0.5 & 0.9 & -0.8 & -11.1 & -3.7 & -12.3 \\
\hline G3 & -0.1 & -0.5 & -0.5 & -1 & -0.8 & -1.1 & -3.7 & -1.8 & -4.5 & 5.9 & 4.7 & 4.2 & 1.8 & 2.6 & 0.3 & -10.1 & -2.6 & -12.7 \\
\hline G4 & 2.7 & 2.1 & 1.9 & 1.9 & 1.9 & 1.3 & -1.3 & 0.7 & -2.7 & 8.8 & 6.7 & 6.1 & 4.6 & 4.6 & 1.9 & -9.2 & -1.8 & -13.5 \\
\hline G5 & 4.5 & 3.3 & 3.2 & 8.3 & 5.8 & 6.1 & 18.2 & 7.9 & -1.5 & 16.9 & 12.7 & 12.1 & 30.4 & 21 & 23.8 & 63.2 & 28.7 & -1 \\
\hline \multicolumn{19}{|c|}{ MSE_JY } \\
\hline G1 & -0.6 & -0.6 & -0.4 & -3.6 & $\begin{array}{l}-2.3 \\
\end{array}$ & -2.5 & -4.4 & -2.1 & -4.1 & 2.4 & 2.2 & 2.4 & -4.8 & -2.1 & \begin{tabular}{|l|}
-3 \\
\end{tabular} & -9.3 & -2.7 & -8.5 \\
\hline G2 & 1 & 0.8 & 0.8 & 0.1 & 0.4 & 0.2 & -2.4 & -0.5 & -2.8 & 4.1 & 3.6 & 3.6 & 0.4 & 1.8 & 0.1 & -10.4 & -3 & -11.7 \\
\hline G3 & 0.4 & 0 & 0 & -0.4 & -0.3 & -0.6 & -3.1 & -1.2 & -4 & 6.8 & 5.5 & 5.1 & 2.8 & 3.5 & 1.2 & -9.2 & -1.8 & -12.2 \\
\hline G4 & 3.2 & 2.6 & 2.4 & 2.5 & 2.4 & 1.8 & -0.7 & 1.2 & -2.2 & 10 & 7.7 & 7.1 & 5.8 & 5.6 & 2.9 & -8.3 & -1 & -12.9 \\
\hline G5 & 5.2 & 3.8 & 3.7 & 9.1 & 6.4 & 6.9 & 20.1 & 8.5 & -1.2 & 18.3 & 13.8 & 13.3 & 32.8 & 22.6 & 25.9 & 69 & 30.8 & -0.6 \\
\hline & & & & & & & & & MSE_J & & & & & & & & & \\
\hline G1 & -2.1 & -1.6 & -1.1 & -4.8 & -3.3 & -4.9 & -5.4 & \begin{tabular}{|c|}
-3.1 \\
\end{tabular} & -4.9 & -0.1 & 0.5 & 1.3 & -6.4 & -3.6 & -4 & -10.4 & \begin{tabular}{|l|}
-3.8 \\
\end{tabular} & -9.5 \\
\hline G2 & -0.7 & -0.3 & -0.1 & -2.4 & -1.3 & -3.8 & -5.3 & -2.6 & -3.8 & 1.5 & 1.8 & 2.4 & -3.8 & -1.4 & -1.2 & -14.9 & -6.6 & -12.8 \\
\hline G3 & -1.4 & -1.1 & -1 & -3.1 & -2.1 & -5 & -6.1 & -3.4 & -5 & 3.7 & 3.5 & 3.5 & -2.3 & -0.1 & -0.5 & -14.5 & -5.9 & -13.4 \\
\hline G4 & 1.2 & 1.3 & 1.1 & -0.5 & 0.4 & -3.5 & -4.1 & -1.2 & -3.5 & 5.9 & 5.2 & 4.8 & -0.5 & 1.3 & 0.3 & -14.7 & -5.8 & -14.3 \\
\hline G5 & 2.6 & 2.3 & 1.9 & 3.2 & 3.2 & -2.9 & -3 & 2.8 & -2.9 & 12.7 & 11 & 9.4 & 14.8 & 14.7 & 13.9 & -3.2 & 11.8 & -2.1 \\
\hline & & & & & & & & & $\mathrm{RR}$ & & & & & & & & & \\
\hline & & & & & & ISE b & ased o & $\mathrm{n} \mathrm{ML}$ & , REN & AL and & $\mathrm{FH} \mathrm{n}$ & nethoc & & & & & & \\
\hline G1 & 1.5 & 0.6 & 0 & 2.4 & 0.7 & 0.1 & 2.4 & 0.5 & 0.2 & 2.8 & 0.8 & 0.1 & 4 & 0.9 & 0.2 & 4.8 & 0.6 & 0.9 \\
\hline G2 & 1.2 & 0.4 & 0 & 1.6 & 0.6 & 0 & 2.2 & 0.7 & 0.1 & 2.5 & 0.7 & 0.1 & 4 & 1.2 & 0 & 7.6 & 2 & 1.5 \\
\hline G3 & 1.3 & 0.5 & 0 & 1.8 & 0.7 & 0 & 2.4 & 0.8 & 0.2 & 2.1 & 0.6 & 0.2 & 3.7 & 1 & 0 & 7.5 & 1.9 & 1.7 \\
\hline G4 & 0.8 & 0.2 & 0 & 1.1 & 0.3 & 0 & 1.7 & 0.5 & 0.1 & 1.8 & 0.5 & 0.3 & 3.4 & 0.9 & 0 & 7.7 & 1.9 & 1.9 \\
\hline G5 & 0.5 & 0.2 & 0.1 & 0.4 & 0.1 & 0.1 & 1.1 & 0 & 0.1 & 0.9 & 0.1 & 1.2 & 0.9 & 0 & 2.8 & 5.7 & 0 & 0 \\
\hline & & & & & & & & & MSE_R & & & & & & & & & \\
\hline G1 & 0 & 0 & 0 & 0.2 & 0.1 & 0.1 & 0.2 & 0.1 & 0.2 & 0 & 0 & 0 & 0.3 & 0.1 & 0.1 & 0.9 & 0.1 & 0.8 \\
\hline G2 & 0 & 0 & 0 & 0 & 0 & 0 & 0.1 & 0 & 0.1 & 0.1 & 0.1 & 0.1 & 0 & 0 & 0 & 1.2 & 0.1 & 1.5 \\
\hline G3 & 0 & 0 & 0 & 0 & 0 & 0 & 0.2 & 0.1 & 0.2 & 0.4 & 0.2 & 0.2 & 0 & 0.1 & 0 & 1 & 0.1 & 1.6 \\
\hline G4 & 0.1 & 0.1 & 0 & 0 & 0 & 0 & 0 & 0 & 0.1 & 0.8 & 0.5 & 0.4 & 0.2 & 0.2 & 0 & 0.9 & 0 & 1.8 \\
\hline G5 & 0.2 & 0.1 & 0.1 & 0.7 & 0.3 & 0.4 & 3.3 & 0.6 & 0 & 2.9 & 1.6 & 1.5 & 9.3 & 4.4 & 5.7 & 39.9 & 8.2 & 0 \\
\hline & & & & & & & & & MSE_J & & & & & & & & & \\
\hline G1 & 0 & 0 & 0 & 0.1 & 0.1 & 0.1 & 0.2 & 0.1 & 0.2 & 0.1 & 0.1 & 0.1 & 0.2 & 0.1 & 0.1 & 0.9 & 0.1 & 0.7 \\
\hline G2 & 0 & 0 & 0 & 0 & 0 & 0 & 0.1 & 0 & 0.1 & 0.2 & 0.1 & 0.1 & 0 & 0 & 0 & 1.1 & 0.1 & 1.4 \\
\hline G3 & 0 & 0 & 0 & 0 & 0 & 0 & 0.1 & 0 & 0.2 & 0.5 & 0.3 & 0.3 & 0.1 & 0.1 & 0 & 0.9 & 0 & 1.5 \\
\hline G4 & 0.1 & 0.1 & 0.1 & 0.1 & 0.1 & 0 & 0 & 0 & 0.1 & 1 & 0.6 & 0.5 & 0.3 & 0.3 & 0.1 & 0.7 & 0 & 1.7 \\
\hline G5 & 0.3 & 0.2 & 0.2 & 0.9 & 0.4 & 0.5 & 4.1 & 0.7 & 0 & 3.4 & 1.9 & 1.8 & 10.8 & 5.1 & 6.7 & 47.6 & 9.5 & 0 \\
\hline & & & & & & & & & MSE_J & & & & & & & & & \\
\hline G1 & 0.1 & 0 & 0 & 0.2 & 0.1 & 0.3 & 0.3 & 0.1 & 0.3 & 0 & 0 & 0 & 0.4 & 0.1 & 0.2 & 1.1 & 0.1 & 0.9 \\
\hline G2 & 0 & 0 & 0 & 0.1 & 0 & 0.1 & 0.3 & 0.1 & 0.1 & 0 & 0 & 0.1 & 0.2 & 0 & 0 & 2.2 & 0.4 & 1.6 \\
\hline G3 & 0 & 0 & 0 & 0.1 & 0.1 & 0.3 & 0.4 & 0.1 & 0.3 & 0.1 & 0.1 & 0.1 & 0.1 & 0 & 0 & 2.1 & 0.4 & 1.8 \\
\hline G4 & 0 & 0 & 0 & 0 & 0 & 0.1 & 0.2 & 0 & 0.1 & 0.4 & 0.3 & 0.2 & 0 & 0 & 0 & 2.2 & 0.3 & 2.1 \\
\hline G5 & 0.1 & 0.1 & 0 & 0.1 & 0.1 & 0.1 & 0.1 & 0.1 & 0.1 & 1.6 & 1.2 & 0.9 & 2.2 & 2.2 & 1.9 & 0.1 & 1.4 & 0 \\
\hline
\end{tabular}


Table 2. Simulated values of the RB and RR of $\widehat{\operatorname{MSE}}\left(\hat{\theta}_{i}^{\mathrm{EB}}\right)$ for $A=1, m=15$, $\psi_{i}$ pattern (I), (II) and (III) under the normal random effects distribution.

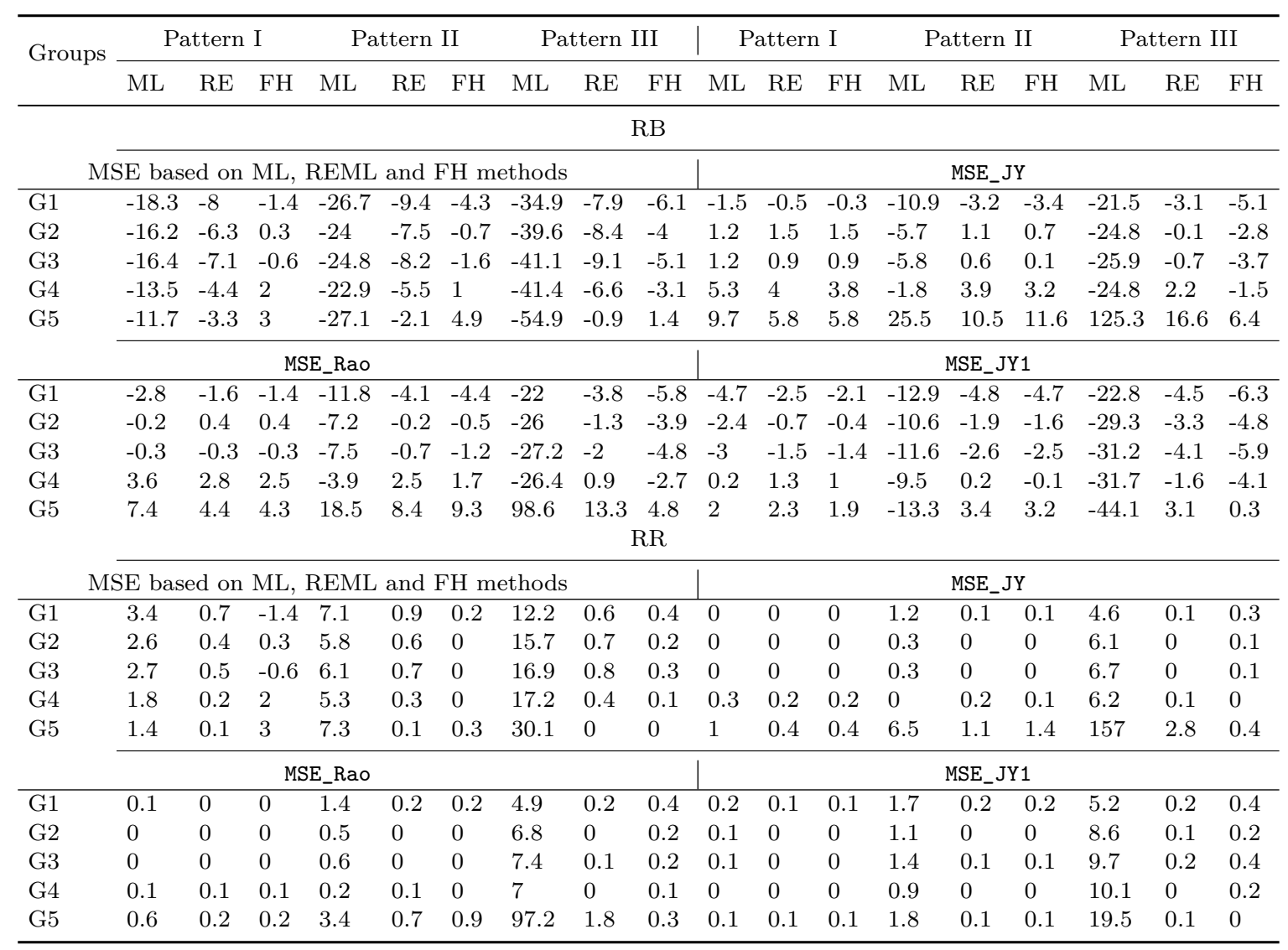




\section{Method}

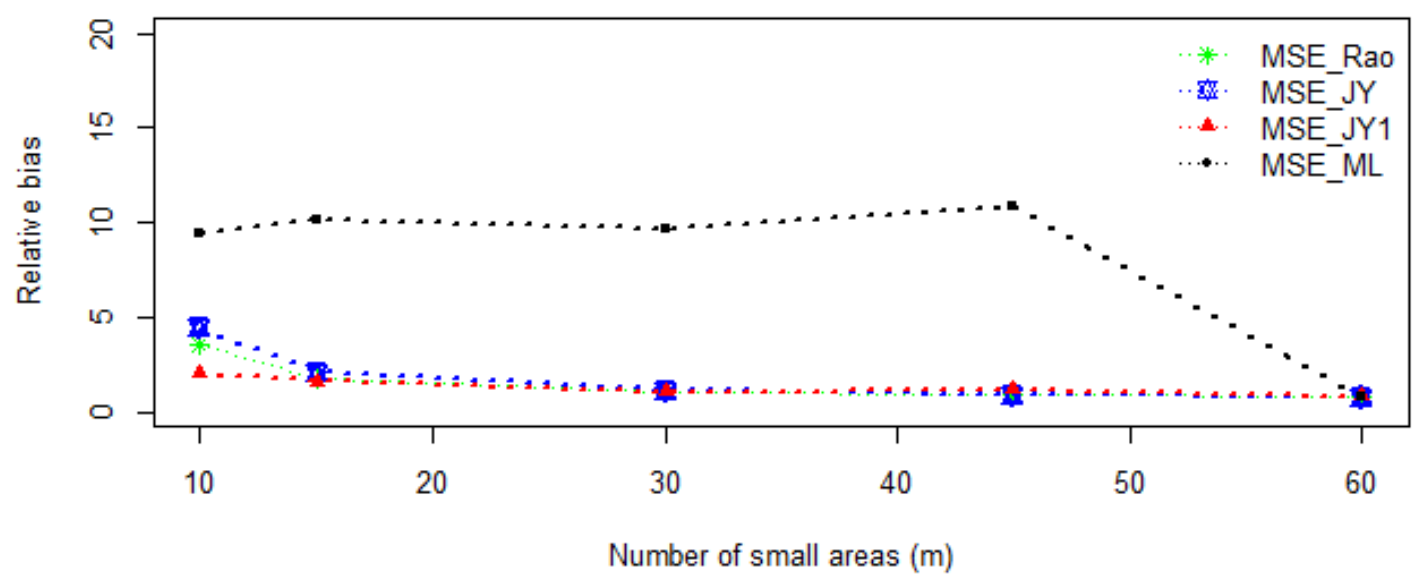

REML Method

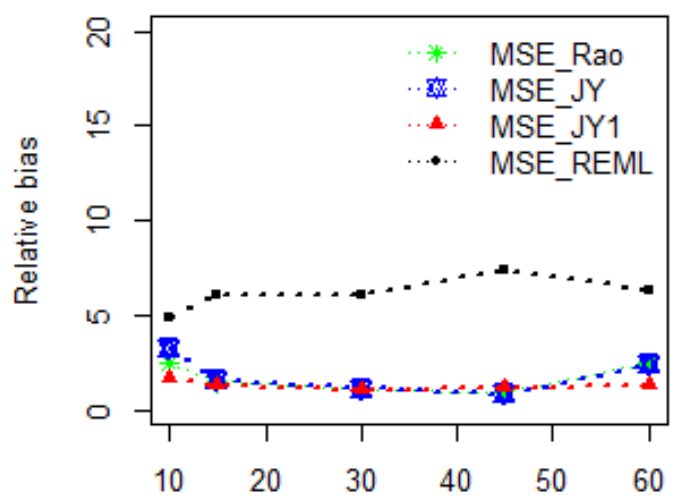

Number of small areas $(\mathrm{m})$
FH Method

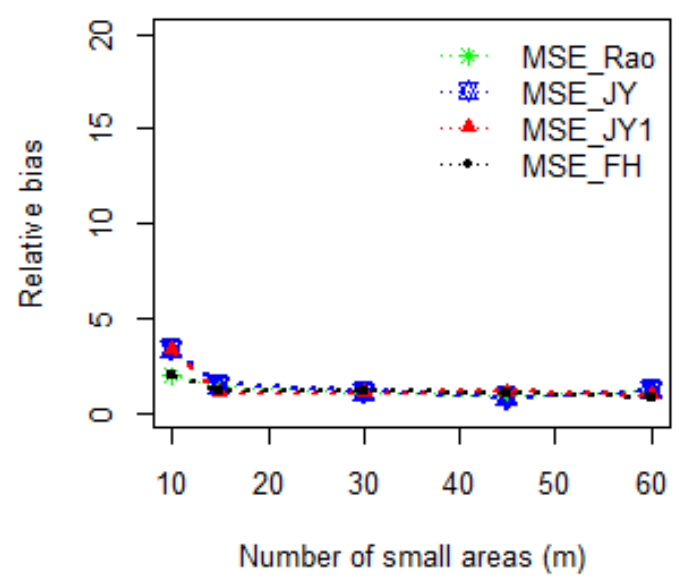

Figure 1. Simulated values of the average of the absolute value of the $\mathrm{RB}$ of $\widehat{\operatorname{MSE}}\left(\hat{\theta}_{i}^{\mathrm{EB}}\right)$ for $A=1$ and $\psi_{i}$ pattern (I) and for the normal random effects distribution. The points are connected with lines for visibility purposes. 
The key findings in Figure 2 are [24]:

- For the $\psi_{i}$ pattern (II), the average RB of all the MSE estimators are less than $15 \%$.

- The average RB of MSE estimators based on the FH method is less than $5 \%$ over all domains. MSE estimators based FH method are nearly unbiased.

- For the ML and REML estimators, MSE_JY1 remains nearly unbiased over all areas.

\section{Method}

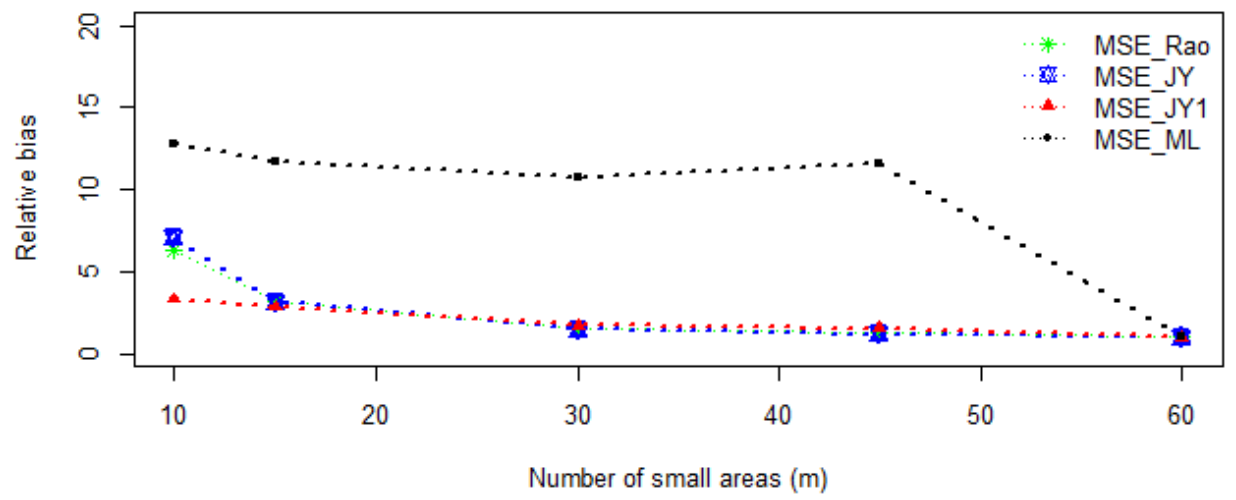

REML Method

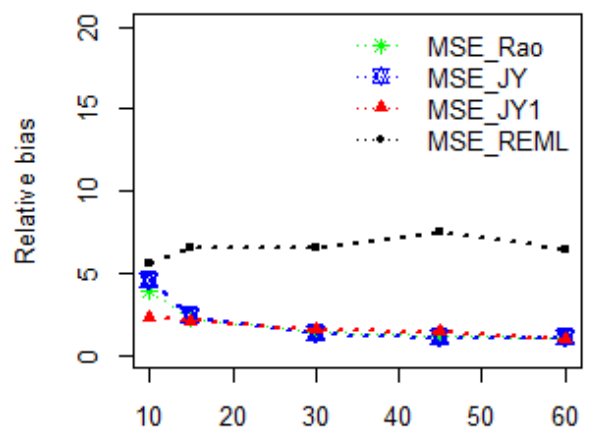

Number of small areas (m)

FH Method

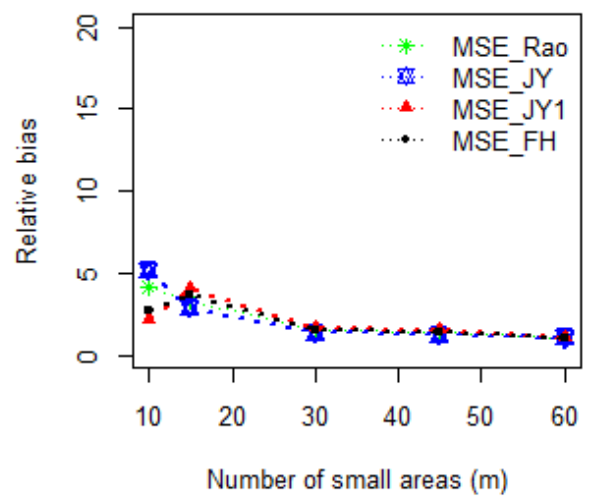

Figure 2. Simulated values of the average of the absolute value of the $\mathrm{RB}$ of $\widehat{\operatorname{MSE}}\left(\hat{\theta}_{i}^{\mathrm{EB}}\right)$ for $A=1$ and $\psi_{i}$ pattern (II) and for the normal random effects distribution. The points are connected with lines for visibility purposes. 
The key findings in Figure 3 are [24]:

- The average RB of MSE estimators decreases as $m$ increases.

- When $m<30$, the average RB of MSE estimators based on MSE_JY1 is small when compared to its competitors for the ML and REML methods.

- MSE estimators are approximately unbiased for the FH method in all cases.

\section{Method}

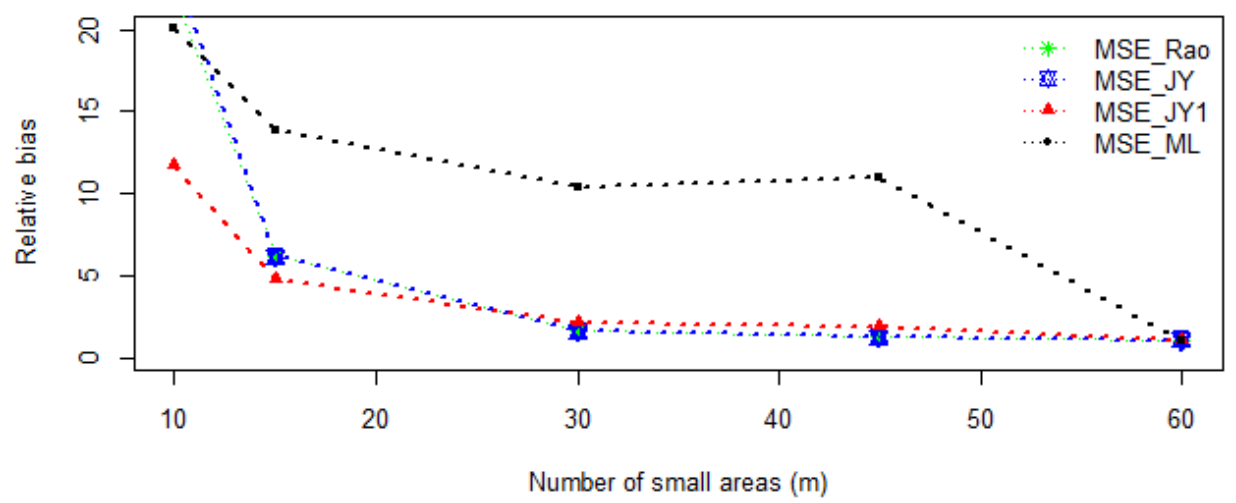

REML Method

FH Method

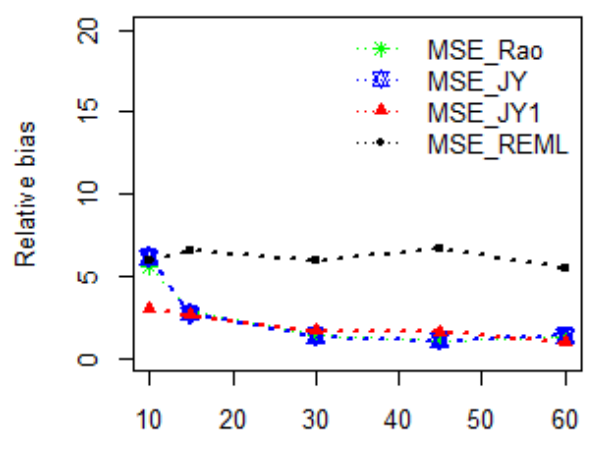

Number of small areas (m)

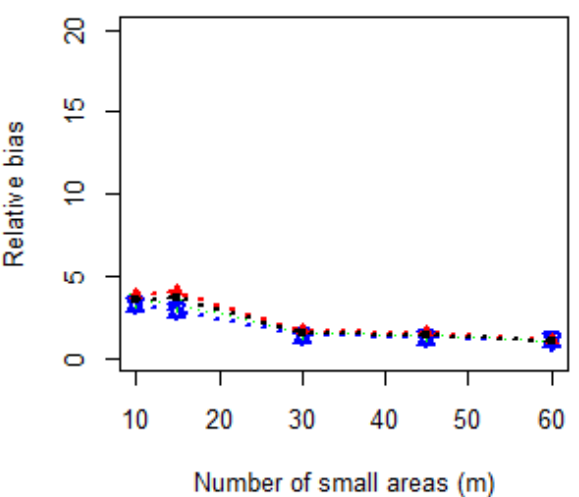

Figure 3. Simulated values of the average of the absolute value of the RB of $\widehat{\operatorname{MSE}}\left(\hat{\theta}_{i}^{\mathrm{EB}}\right)$ for $A=1$ and $\psi_{i}$ pattern (III) and for the normal random effects distribution. The points are connected with lines for visibility purposes. 
3.1.4. Simulation results of the coverage probability. This section explored the CPs of the confidence intervals given in equations (3.6-3.11). The MSEs and CPs from the simulation study for the normal distribution and $m=20$ are summarized in Table 3. As shown in this table, the corrected confidence intervals such as $\mathrm{I}_{i}^{\mathrm{FH}}, \mathrm{I}_{i}^{\mathrm{Rao}}, \mathrm{I}_{i}^{\mathrm{JY}}$ and $\mathrm{I}_{i}^{\mathrm{JY} 1}$ have larger CPs than the uncorrected (naive) confidence intervals such as $\mathrm{I}_{i}^{\mathrm{PR}}$ and $\mathrm{I}_{i}^{\mathrm{Cox}}$, indicating that the coverage is quite accurate [24]. Figure 4 also shows the CPs of the confidence intervals (3.6-3.11) over a range of $m$.

Table 3. Simulated values of $\widehat{\mathrm{MSE}}\left(\hat{\theta}_{i}^{\mathrm{EB}}\right)$ multiplied by 100 and $\mathrm{CPs}$ of $\mathrm{I}_{i}^{\mathrm{Cox}}, \mathrm{I}_{i}^{\mathrm{PR}}$, $\mathrm{I}_{i}^{\mathrm{FH}}, \mathrm{I}_{i}^{\mathrm{Rao}}, \mathrm{I}_{i}^{\mathrm{JY}}$ and $\mathrm{I}_{i}^{\mathrm{JY} 1}$ for $A=1, m=20, \psi_{i}$ pattern II and for the normal random effects distributions.

\begin{tabular}{cccccccc}
\hline & & \multicolumn{6}{c}{ Coverage probability } \\
\cline { 3 - 8 } Groups & MSE & $\mathrm{I}_{i}^{\text {Cox }}$ & $\mathrm{I}_{i}^{\mathrm{PR}}$ & $\mathrm{I}_{i}^{\mathrm{FH}}$ & $\mathrm{I}_{i}^{\text {Rao }}$ & $\mathrm{I}_{i}^{\mathrm{JY}}$ & $\mathrm{I}_{i}^{\mathrm{JY} 1}$ \\
\hline $\mathrm{G} 1$ & 70.4 & 90.6 & 93.1 & 93.8 & 94.0 & 93.7 & 93.8 \\
\hline $\mathrm{G} 2$ & 38.7 & 91.8 & 94.2 & 94.5 & 94.6 & 94.5 & 94.7 \\
\hline $\mathrm{G} 3$ & 34.3 & 91.9 & 94.4 & 94.8 & 94.7 & 94.7 & 94.7 \\
\hline $\mathrm{G} 4$ & 29.4 & 92.1 & 94.6 & 94.8 & 94.8 & 94.6 & 95.0 \\
\hline $\mathrm{G} 5$ & 17.2 & 93.1 & 95.0 & 95.2 & 95.1 & 95.3 & 95.0 \\
\hline
\end{tabular}

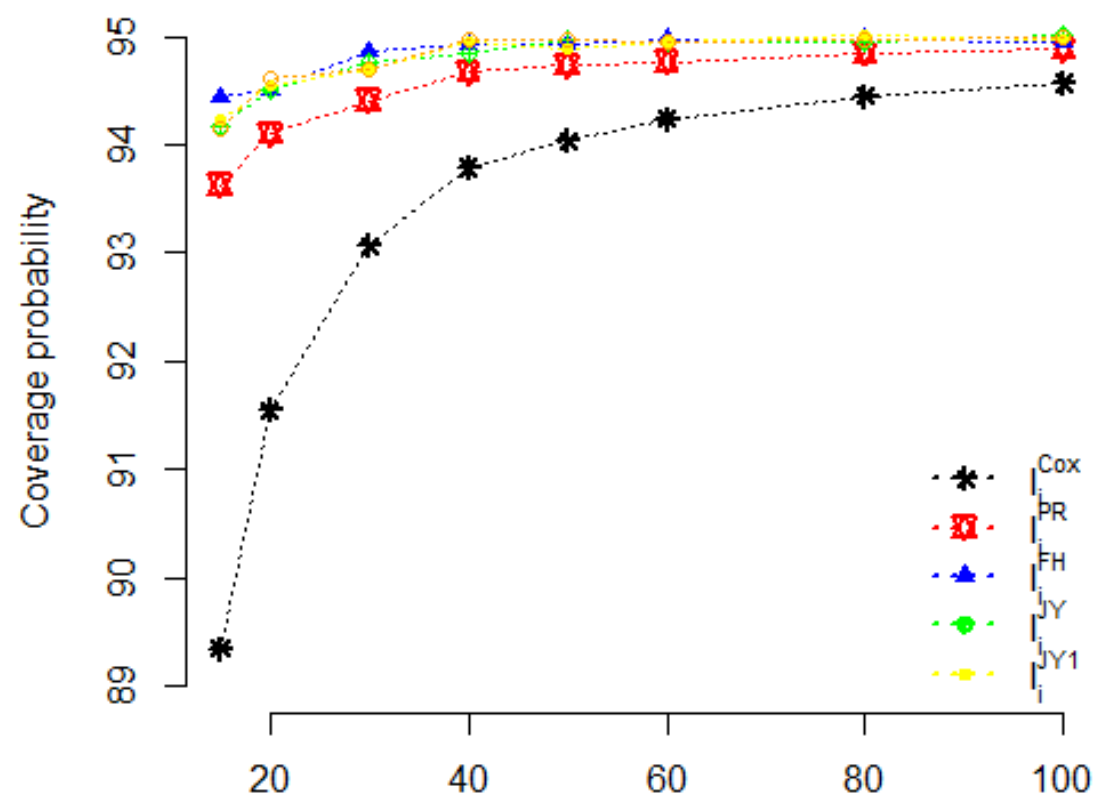

Number of small areas, $m$

Figure 4. Simulated values of the $\mathrm{CPs}$ of $\mathrm{I}_{i}^{\mathrm{Cox}}, \mathrm{I}_{i}^{\mathrm{PR}}, \mathrm{I}_{i}^{\mathrm{FH}}, \mathrm{I}_{i}^{\mathrm{Rao}}, \mathrm{I}_{i}^{\mathrm{JY}}$ and $\mathrm{I}_{i}^{\mathrm{JY} 1}$ for nominal $95 \%$ CIs for $\psi_{i}$ pattern III and FH method. The points are connected with lines for visibility purposes. 
3.1.5. Discussion of simulation results. In this section, we discuss results from the simulation studies of the area-specific MSE estimators. We investigate the performances of the proposed area-specific MSE estimators using simulation studies. As shown in Table 1 , in terms of RB and RR the MSE estimators based on the ML, REML and FH behave similarly for the pattern (I), with no particular one emerging as clearly better than the other three. When $m=15$, estimators based on the FH method have a very small bias for all variance patterns. For the same pattern, the bias of MSE estimators are generally positive and thus accumulative at higher levels of aggregation. The bias of the MSE_JY1 estimator is smaller than MSE_Rao and MSE JY estimators for the ML and REML methods. All the estimators have small biases and risks for the case where $\hat{A}=\hat{A}_{F H}$ and the biases are generally smaller than the biases obtained when $\hat{A}=\hat{A}_{M L}$ for all variance patterns, particularly for the smaller variance $\psi_{i}$. The bias and risk of all the estimators are less than $7 \%$ when $\hat{A}=\hat{A}_{F H}$ for both patterns (II) and (III) are negligible for group G5. This result is in agreement with the previous results of [6] and [5]. Thus, the FH method performed better than the ML and REML methods for patterns (II) and (III). The RR of MSE_Rao, MSE_JY and MSE_JY1 are nearly zero for the ML, REML and FH methods for all patterns. In terms of RR, for the pattern (I), all the MSE estimators are robust. In addition, in terms of RR, all the MSE estimators are robust for all patterns [24].

Results under the Laplace distribution: the $\mathrm{RB}$ and $\mathrm{RR}$ are generally higher than the $\mathrm{RB}$ and $\mathrm{RR}$ when the random effects are generated from the normal distributions. The MSE estimator based on the ML method has a large negative bias. For example, the RB of the MSE estimators are $-23.8 \%, 63.2 \%, 69 \%$ and $-3.2 \%$ for MSE_ML, MSE_Rao, MSE_JY and MSE_JY1, respectively when $\hat{A}=\hat{A}_{M L}$ for pattern (III) and $\psi_{i}=0.1$ (small sampling error variance). All the methods are sensitive to the deviation from normality of the random effects distribution considered in the simulation for all patterns with $\psi_{i}=0.1$ except the FH method for pattern (III). The RB of MSE_JY1 is smaller when $\hat{A}=\hat{A}_{F H}$ for the pattern (III) and $\psi_{i}=0.1$. The RR of MSE estimators based on the $\mathrm{FH}$ method are less than $7 \%$ in all cases. In summary, MSE estimators based on the REML and FH methods are robust [24].

For the FH model incorporating covariates, the RB and RR of MSE estimators are presented in Table 2. For the simulation exercise with covariates MSE estimators based on ML methods are biased for all variance patterns. The RB of MSE estimators based on ML method are $-54.9 \%, 98.6 \%, 125.3 \%$ and $-44.1 \%$ for MSE_ML, MSE_Rao, MSE_JY and MSE_JY1 respectively for G5 (the smallest variance in pattern (III)). MSE_JY1 performs well for the ML, REML and FH methods. The RR of MSE_ML, MSE_Rao, MSE_JY and MSE_JY1 are 30.1\%, 97.2\%, 157\% and 19.5\%, respectively for G5. For pattern (II), the average RR of MSE_ML, MSE_Rao, MSE_JY and MSE_JY1 are 6.32\%, $1.22 \%$, $1.66 \%$ and $1.38 \%$ respectively. However, the RR of MSE estimators based on the REML and FH methods are negligible (less than 5\%) for all patterns indicating the robustness of these methods. Thus, it is recommended to use MSE estimators based on these two methods [24].

Lastly, the simulation results in Table 3 shows the estimated CPs of confidence intervals for $\theta_{i}$ under the normal random effects distribution for $m=20$. The coverage accuracy of the confidence intervals based on the area specific MSEs: $\mathrm{I}_{i}^{\mathrm{Rao}}, \mathrm{I}_{i}^{\mathrm{JY}}$ and $\mathrm{I}_{i}^{\mathrm{JY} 1}$ are larger than the coverage accuracy of the naive confidence intervals: $\mathrm{I}_{i}^{\text {Cox }}$ and $\mathrm{I}_{i}^{\mathrm{PR}}$. In addition, the coverage accuracy of the corrected confidence interval based on the non area specific MSE (i.e., $\mathrm{I}_{i}^{\mathrm{FH}}$ ) is comparable with the confidence intervals based on the area specific MSEs ( $\mathrm{I}_{i}^{\mathrm{Rao}}, \mathrm{I}_{i}^{\mathrm{JY}}$ and $\mathrm{I}_{i}^{\mathrm{JY} 1}$ ). In other words, all the corrected confidence intervals given in equations $(3.8-3.11)$ meet the nominal coverage rate better than the naive confidence intervals given in equations (3.6 - 3.7). Figure 4 shows that with the increase of $m$ the CPs 
of all the confidence intervals increase. In other words, all the above confidence intervals based on the area specific MSEs gets better and the percentage difference between their CPs are negligible, supporting asymptotic theory. There is very little difference observed by using any of the confidence intervals and MSEs when $m \geq 60$. These findings are consistent with the findings in the above related literatures [14,22,23].

\subsection{Application Results}

In this section, we illustrate the MSE estimators in an application to a real data set relating to the estimation of the percentage of food expenditure and poverty measures in all zones of Ethiopia. This helps us to examine the performance of the MSE estimators using real data sets.

3.2.1. Data sources. The $2010 / 11$ household consumption expenditure survey (HCES) and the 2007 housing and population census of Ethiopia were used to study the performances of the MSE estimators. The HCES was conducted by the government of statistical agency of Ethiopia. The survey includes Rural, Major urban centers, and Other urban centers categories. In the first two categories, the two stage cluster sampling method was implemented by considering enumeration areas as the primary sampling units and 12 households as the secondary sampling units. In the third category, a stratified three stage cluster sampling was considered by taking urban centers as primary sampling units, enumeration areas as secondary units and households as a third stage sampling units. A total of 27,830 households were successfully covered by the survey at the country level. In order to fill the possible gap in the survey data, we used the auxiliary information from the 2007 Ethiopian census data. This can be achieved by borrowing strength from related zones, based on a model linking all zones using the FH model. The HCES data are available for 86 zones, regional towns, Addis Ababa sub cities (Kifle Ketemas) and Dire Dawa sub city in Ethiopia. These are the second level administrative divisions in Ethiopia, and we consider each zone as a small area, namely $m=86$. For the $i^{\text {th }}$ zone, data of $n_{i}$ are available in 2011.

3.2.2. Example 1: percentage of food expenditure. For $i=1,2, \cdots, m, y_{i}$ is the annual household consumption expenditure for households in the $i^{\text {th }}$ zone. The simple analytical expression of the percentage of food expenditure $y_{i}$ for the $i^{\text {th }}$ zone is given as

$$
y_{i}=\left(\frac{\mathrm{FE}_{i}}{\mathrm{TE}_{i}}\right) * 100 \%, \quad i=1,2, \cdots, m,
$$

where total expenditure (TE) refers to expenses for accommodation, food, purchases, travel, leisure activities and miscellaneous expenditure, while food expenditure (FE) refers expenses on food. The Akaike information criterion (AIC), Bayesian information criterion (BIC) and log-likelihood function were used to choose regressor variables and propose the following linear mixed model:

$$
y_{i}=x_{1 i} \beta_{1}+x_{2 i} \beta_{2}+x_{3 i} \beta_{3}+x_{4 i} \beta_{4}+x_{5 i} \beta_{5}+x_{6 i} \beta_{6}+v_{i}+e_{i}, i=1, \ldots, m,
$$

where $x_{1}$ denotes men, $x_{2}$ denotes age category less than or equal to $30, x_{3}$ denotes single marital status, $x_{4}$ denotes greater than 12 years of schooling, $x_{5}$ denotes employed and $x_{6}$ denotes household size less than or equal to 5 . Then, the estimates of the parameters are given by $\hat{A}=28.79$, and $\hat{\boldsymbol{\beta}}(\hat{A})=\left(\hat{\beta}_{1}, \hat{\beta}_{2}, \hat{\beta}_{3}, \hat{\beta}_{4}, \hat{\beta}_{5}, \hat{\beta}_{6}\right)=(14.375,-5.8656,8.788,-69.1976$, $29.5893,-0.3843)$. It is interesting to note that the percentage of food expenditure decreases when age category less than or equal to 30 , greater than 12 years of schooling and household size less than or equal to 5 increases.

Now we give the MSEs of the average percentage of food expenditure for the $i^{\text {th }}$ zone, namely, $\theta_{i}=x_{1 i} \beta_{1}+x_{2 i} \beta_{2}+x_{3 i} \beta_{3}+x_{4 i} \beta_{4}+x_{5 i} \beta_{5}+x_{6 i} \beta_{6}+v_{i}$ for $i=1, \cdots, m$. The average RB and RR of MSE estimators shown in equation (3.5) and the average width 
of prediction intervals based on the $\mathrm{FH}$ moment estimator are computed to study the performance of the MSE estimators using real data sets. The FH moment estimator was chosen to estimate the variance of the random effect since it does not require normality assumptions. It was also performed better than the other competitive methods through a simulation study. In addition to RB and RR, the average width of the following prediction intervals are computed using the following equations (see [16]):

$$
\begin{aligned}
\mathrm{CI}_{i}^{\mathrm{FH}} & =\hat{\theta}_{i}^{\mathrm{EB}} \mp Z_{\alpha / 2} \sqrt{\mathrm{MSE}_{-} \mathrm{FH}_{i}} . \\
\mathrm{CI}_{i}^{\mathrm{Rao}} & =\hat{\theta}_{i}^{\mathrm{EB}} \mp Z_{\alpha / 2} \sqrt{\mathrm{MSE}_{-} \mathrm{Rao}_{i}} . \\
\mathrm{CI}_{i}^{\mathrm{JY}} & =\hat{\theta}_{i}^{\mathrm{EB}} \mp Z_{\alpha / 2} \sqrt{\mathrm{MSE}_{-} \mathrm{JY}_{i}} . \\
\mathrm{CI}_{i}^{\mathrm{JY} 1} & =\hat{\theta}_{i}^{\mathrm{EB}} \mp Z_{\alpha / 2} \sqrt{\mathrm{MSE}_{-} \mathrm{JY}_{i}} .
\end{aligned}
$$

We used the average $\mathrm{RB}$, average $\mathrm{RR}$ and the average width of prediction intervals to study the performance of the MSE estimators. The MSE estimators based on the FH method were used to obtain the $\mathrm{RB}, \mathrm{RR}$ and width of prediction intervals. Table 4 shows the average $\mathrm{RB}, \mathrm{RR}$ and width of prediction intervals for the percentage of food expenditure over the $m=15,30,45,60,86$ sampled Ethiopian zones. From this table, we observe that the average RB, RR and width of prediction intervals for the MSE estimators based on the JY equation (2.13) and JY1 equation (2.14) are slightly smaller than the one based on equation (2.11) and equation (2.12), indicating that MSE_JY and MSE_JY1 estimators provide better performance.

Table 4. Mean relative bias (RB), mean relative risk (RR) and mean width of prediction intervals for percentage of food expenditure using the 2010/11 HCES and 2007 population and housing census of Ethiopia

\begin{tabular}{c|c|cccc}
\hline Number of sampled areas (m) & & MSE_FH & MSE_JY1 & MSE_JY & MSE_Rao \\
\hline \multirow{3}{*}{15} & RB & 0.749 & 0.454 & 0.649 & 0.737 \\
& RR & 0.006 & 0.005 & 0.009 & 0.005 \\
\multirow{3}{*}{30} & Width of CI & 5.679 & 5.663 & 5.674 & 5.679 \\
& RB & 0.494 & 0.398 & 0.533 & 0.490 \\
& RR & 0.002 & 0.004 & 0.006 & 0.002 \\
\multirow{2}{*}{45} & Width of CI & 5.646 & 5.641 & 5.648 & 5.646 \\
& RB & 0.282 & 0.244 & 0.327 & 0.281 \\
\multirow{3}{*}{60} & RR & 0.001 & 0.002 & 0.003 & 0.001 \\
& Width of CI & 5.679 & 5.677 & 5.682 & 5.679 \\
& RB & 0.193 & 0.174 & 0.231 & 0.192 \\
& RR & 0.000 & 0.001 & 0.002 & 0.000 \\
& Width of CI & 5.677 & 5.676 & 5.679 & 5.677 \\
& RB & 0.177 & 0.165 & 0.198 & 0.176 \\
& RR & 0.000 & 0.001 & 0.002 & 0.000 \\
& Width of CI & 5.636 & 5.635 & 5.637 & 5.636 \\
\hline
\end{tabular}

3.2.3. Example 2: incidence of total poverty (Headcount Ratio), HCO. Consider a finite population $D$ of size $N$ partitioned into $m$ areas $D_{i}, i=1, \ldots, m$. Let $S \subset D$ be a sample drawn from the population and let $S_{i}=S \cap D_{i}$ be the sample from area $i$. The poverty incidence, gap and severity index are measured using the well-known FGT measures [10]. Using this method, an approximately design unbiased survey based direct estimator of $P_{\alpha i}$ for the $i^{t h}$ zone is given by (see [20], [18])

$$
\hat{P}_{\alpha i}=\frac{1}{\hat{N}_{i}} \sum_{j \in S_{i}} w_{i j}\left(\frac{Z-E_{i j}}{Z}\right)^{\alpha} I\left(E_{i j}<Z\right) ; \quad i=1, \ldots, m, j=1, \ldots, N_{i}, \alpha=0,1,2,
$$


where $\hat{N}_{i}=\sum_{j \in S_{i}} w_{i j}, w_{i j}$ be the sampling weight of individual $j$ from sampled area $i$ and $I\left(E_{i j}<Z\right)=1$ if $E_{i j}<Z$ (person under poverty) and $I\left(E_{i j}<Z\right)=0$ otherwise (person not under poverty). The FGT poverty indicators $\hat{P}_{\alpha i}$ are defined as poverty incidence (or Headcount Ratio) if $\alpha=0$, poverty gap if $\alpha=1$ and poverty severity if $\alpha=2$. The FH model can be used with

$$
\theta_{i}=P_{\alpha i}, \quad y_{i}=\hat{P}_{\alpha i}, \quad \psi_{i}=\operatorname{Var}\left(\hat{P}_{\alpha i}\right)=\hat{V}\left(y_{i}\right)
$$

where the design-based variances of these estimators is given by (see [8])

$$
\hat{V}\left(y_{i}\right)=\frac{1}{\hat{N}_{i}^{2}} \sum_{j \in S_{i}} w_{i j}\left(w_{i j}-1\right)\left(E_{i j}-y_{i}\right)^{2}
$$

We use the generalized variance function (GVF) to smooth out the uncertainty of the design based variance estimate equation (3.18). The GVF was first introduced by [9] in a complex sample survey setting in order to motivate the sampling error component of their two level Bayesian model. Then the estimated GVF is given by

$$
\widehat{G V F}=\exp \left(\frac{\hat{\sigma}^{2}}{2}\right) \exp \left(\hat{b}_{0}+\hat{b}_{1} y_{i}\right)
$$

where $\hat{b}_{0}$ and $\hat{b}_{1}$ are the least squares estimates from the log-linear regression model $\log \left(\hat{V}\left(y_{i}\right)\right)=b_{0}+b_{1} y_{i}+\epsilon_{i}$ and $\epsilon_{i} \stackrel{i i d}{\sim} N\left(0, \sigma^{2}\right), i=1, \ldots, m$. If we ignore the correction term in the GVF method, underestimation of the true variances will be occurred $([21],[8])$.

The proportion of individuals under poverty in the $i^{\text {th }}$ zone is called headcount ratio or poverty incidence $\left(P_{0 i}\right.$, this ratio corresponds when $\left.\alpha=0\right)$. We use the 2010/11 HCES and the 2007 population and housing census of Ethiopia to estimate poverty headcount index for the zones, regional towns, Addis Ababa sub cities and Dire Dawa sub city in Ethiopia. The response variable is the estimate of poverty measures based on the 2010/11 HCES data. An Ethiopian household is considered to be living in poverty when its consumption expenditure is below the poverty line of 3,781 birrs (Ethiopian currency) per adult per year $([15])$.

Similar to the percentage of food expenditure case in section (3.2.2.), the AIC, BIC and log-likelihood function were used to propose the following linear mixed model

$$
y_{i}=x_{1 i} \beta_{1}+x_{2 i} \beta_{2}+x_{3 i} \beta_{3}+x_{4 i} \beta_{4}+x_{5 i} \beta_{5}+x_{6 i} \beta_{6}+v_{i}+e_{i}, i=1, \ldots, m,
$$

where $x_{1}$ denotes women, $x_{2}$ denotes age category less than or equal to $30, x_{3}$ denotes married, $x_{4}$ denotes greater than 12 years of schooling, $x_{5}$ denotes employed and $x_{6}$ denotes household size less than or equal to 5 . Then, the estimates of the parameters are given by $\hat{A}=23.78$, and $\hat{\boldsymbol{\beta}}(\hat{A})=\left(\hat{\beta}_{1}, \hat{\beta}_{2}, \hat{\beta}_{3}, \hat{\beta}_{4}, \hat{\beta}_{5}, \hat{\beta}_{6}\right)=(7.7328,-0.6766,-0.1154,-0.4565,2.6392$, $-1.0076)$. Here it is also interesting to see poverty decreases when the household has family of less than 5, married, greater than 12 years schooling, and employed. Now we obtain the RB, RR (3.5) and width of prediction intervals (3.15) to assess the performances of the different MSE estimators. These results are presented in Table 5. From this table, we can easily observe that MSE_JY1 performed slightly better than the other three MSEs considered in this paper under the FH method. 
Table 5. Mean relative bias (RB), mean relative risk (RR) and mean width of prediction intervals for poverty measures using the 2010/11 HCES and 2007 population and housing census of Ethiopia

\begin{tabular}{c|c|cccc}
\hline Number of sampled areas (m) & & MSE_FH & MSE_JY1 & MSE_JY & MSE_Rao \\
\hline \multirow{3}{*}{15} & RB & 27.718 & 18.458 & 21.383 & 27.27 \\
& RR & 8.948 & 5.984 & 7.389 & 8.675 \\
& Width of CI & 0.610 & 0.565 & 0.585 & 0.607 \\
\hline \multirow{2}{*}{30} & RB & 8.004 & 6.257 & 6.78 & 7.917 \\
& RR & 0.650 & 0.864 & 0.990 & 0.636 \\
& Width of CI & 0.568 & 0.561 & 0.564 & 0.567 \\
\hline \multirow{2}{*}{45} & RB & 4.430 & 3.683 & 3.917 & 4.397 \\
& RR & 0.197 & 0.329 & 0.366 & 0.194 \\
& Width of CI & 0.578 & 0.576 & 0.577 & 0.578 \\
\hline \multirow{2}{*}{60} & RB & 2.483 & 2.166 & 2.301 & 2.469 \\
& RR & 0.062 & 0.126 & 0.147 & 0.061 \\
& Width of CI & 0.620 & 0.619 & 0.62 & 0.620 \\
\hline \multirow{2}{*}{86} & RB & 1.724 & 1.592 & 1.681 & 1.717 \\
& RR & 0.030 & 0.062 & 0.067 & 0.030 \\
& Width of CI & 0.618 & 0.618 & 0.619 & 0.618 \\
\hline
\end{tabular}

We also examined the performance of the proposed methods over a range of $m$ (see Tables 4 and 5). The average RB, average $\mathrm{RR}$ and the average width of prediction intervals were obtained for the number of areas such as $\mathrm{m}=15,30,45,60$ and 86. Our proposed methods (i.e., MSE_JY and MSE_JY1) have slightly lower RB and RR especially for lower sampled small areas, $m$. When the number of sampled areas, $m$, are small, the area specific MSE estimators perform better when compared to the non-area specific counterparts. When $m \geq 45$, the percentage difference between average $\mathrm{RB}$, average $\mathrm{RR}$ and the average width of prediction intervals are negligible.

\section{Conclusions}

The results of this study indicate that the REML and FH methods perform consistently better than the other methods for normal random effects distribution. For Laplace random effects distribution, FH performs better than other methods. In terms of RB and RR, MSE_JY1 performs even better than the existing MSE estimators based on the ML and REML methods. The difference, however, gets smaller as the number of areas increases for all variance patterns. Thus, it is advisable to use MSE estimators based on REML and FH methods for normal random effects distribution; the FH method for Laplace random effects distribution. The usefulness of the proposed methods was illustrated under the FH model using real data sets. We chose the 2010/11 HCES and the 2007 census data sets to demonstrate how the methods work. The RB, RR and width of prediction intervals based on the FH method were computed to assess the reliability of the MSE estimates.

Acknowledgment. The author would like to thank the editor and reviewer(s) for their valuable comments and suggestions that led to an improved version of this paper. The author would also like to thank the Central Statistical Agency of Ethiopia for the data used in the research. Furthermore, the part of the simulation results for the manuscript are from my $\mathrm{PhD}$ thesis that has been submitted to the University of the Witwatersrand. The findings of this paper reflect the opinions of the author. 


\section{References}

[1] R. Ananya, Empirical and Hierarchical Bayesian Methods with Applications to Small Area Estimation, PhD Dissertation, University of Florida, 2007.

[2] G.E. Battese, R.M. Harter and W.A. Fuller, An error components model for prediction of county crop areas using survey and satellite data, J. Amer. Statist. Assoc. 83, 28-36, 1988.

[3] A. Bianchi, E. Fabrizi, N. Salvati and N. Tzavidis, Estimation and testing in $M$ quantile regression with applications to small area estimation, Int. Stat. Rev. 86, 541-570, 2018.

[4] D. R. Cox, A Prediction intervals and empirical Bayes confidence intervals. In Perspectives in probability and statistics, Papers in honor of M.S. Bartlett (ed. J. Gani), 47-55, Academic Press, London, 1975.

[5] G.S. Datta and P. Lahiri, A unified measure of uncertainty of estimated best linear unbiased predictors in small area estimation problems, Statist. Sinica 10, 613-627, 2000.

[6] G.S. Datta, J.N.K. Rao, and D.D. Smith, On measuring the variability of small area estimators under a basic area level model, Biometrika 92, 183-196, 2005.

[7] G.S. Datta, T. Kubokawa, I. Molina, and J.N.K. Rao, Estimation of mean squared error of model-based small area estimators, Test 20, 367-388, 2011.

[8] M.D. Esteban, D. Morales, A. Perez and L. Santamaria, Small area estimation of poverty proportions under area-level time models, Comput. Statist. Data Anal. 56, 2840-2855, 2012.

[9] R. E. Fay and R.A. Herriot, Estimates of income for small places: An application of James-Stein procedure to census data, J. Amer. Statist. Assoc. 74, 269-277, 1979.

[10] J. Foster, J. Greer, and E. Thorbecke, A class of decomposable poverty measures, Econometrica 52, 761-766, 1984.

[11] J. Jiang and P. Lahiri, Mixed model prediction and small area estimation, Test 15, 1-96, 2006.

[12] T. Kubokawa and B. Nagashima, Parametric bootstrap methods for bias correction in linear mixed models, J. Multivariate Anal. 106, 1-16, 2012.

[13] H. Li, Small area estimation: An empirical best linear unbiased predictor approach. $\mathrm{PhD}$ dissertation, University of Maryland, United States, 2007.

[14] H. Li and P. Lahiri, An adjusted maximum likelihood method for solving small area estimation problems, J. Multivariate Anal. 101, 882 - 892, 2010.

[15] MoFED, Ethiopia's progress towards eradicating poverty: an interim report on poverty analysis study, Development Planning and Research Directorate, Addis Ababa, Ethiopia, 2012.

[16] I. Molina, J.N.K. Rao and G.S. Data, Small area estimation under a Fay-Herriot model with preliminary testing for the presence of random effects, Survey Methodology 41, 1-19, 2015.

[17] N.G.N. Prasad and J. N. K. Rao, The estimation of the mean squared error of small area estimators, J. Amer. Statist. Assoc. 85, 163-171, 1990.

[18] M. Pratesi, Analysis of poverty data by small area estimation, John Wiley and Sons, 2015.

[19] J.N.K. Rao, EB and EBLUP in small area estimation in S. E. Ahmed and N. Reid (Eds.); Empirical Bayes and Likelihood Inference, Lecture Notes in Statistics 148, New York: Springer, 33-43, 2001.

[20] J.N.K Rao and I. Molina, Small Area Estimation. John Wiley and Sons, Inc., New York, 2015.

[21] L.P. Rivest and E. Belmonte, A Conditional Mean Squared Error of Small Area Estimators, Survey Methodology 26, 79-90, 2000. 
[22] Y.A. Shiferaw and J.S. Galpin, Area specific confidence intervals for a small area mean under the Fay-Herriot model, J. Iran. Stat. Soc. 15, 1-44, 2016.

[23] Y. Shiferaw and J. Galpin, A corrected confidence interval for a small area parameter through the weighted estimator under the basic area level model, J. Iran. Stat. Soc. 18, 17-51, 2019.

[24] Y.A. Shiferaw and J.S. Galpin, Improved confidence intervals for a small area mean under the Fay-Herriot model, Accessed from wiredspace.wits.ac.za.

[25] Q. Yanping, G.D. Meeden and B. Zhang, An objective stepwise Bayes approach to small area estimation, J. Stat. Comput. Simul. 85, 1474-1494, 2015.

[26] M. Yoshimori and P. Lahiri, A second-order efficient empirical Bayes confidence interval, Ann. Statist. 42, 1233-1261, 2014. 